\title{
The influence of Schizotypy on Event- Related Oscillations in Sensory Gating during early Infant Development.
}

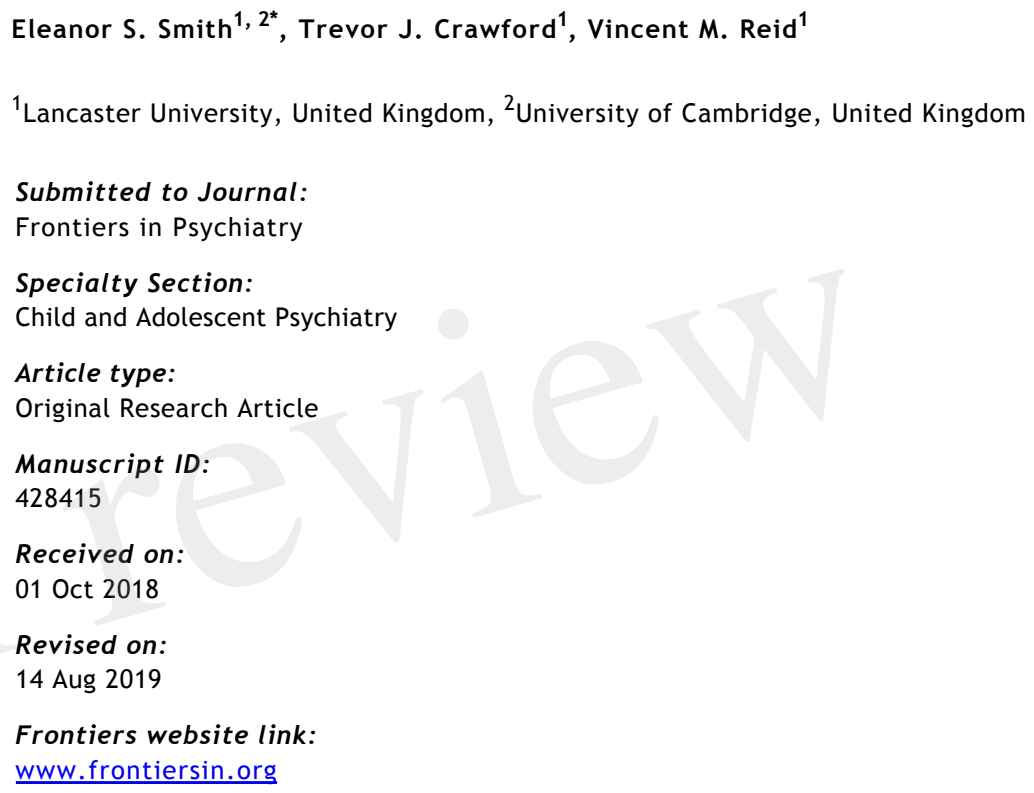




\section{Conflict of interest statement}

The authors declare that the research was conducted in the absence of any commercial or financial relationships that could be construed as a potential conflict of interest

\section{Author contribution statement}

Eleanor Smith carried out recruitment, testing, pre-processing, and time-frequency analysis. She also ran data analysis and wrote the paper.

Trevor Crawford and Vincent Reid both acted as Supervisors throughout the research and provided their input on paper development.

\section{Keywords}

EEG, Event-related oscillations (EROs), Sensory Gating, Infancy, schizotypy

\section{Abstract}

Word count: $\quad 215$

Maternal personality is known to influence childhood risk factors for mental health. More specifically, maternal psychopathologies, such as those on the schizophrenia-spectrum have been associated with P50 sensory gating abilities. Schizotypy is a personality dimension within the general population elevated among schizophrenia-spectrum patients and their first-degree relatives. Sensory gating is the pre-attentional habituation of responses distinguishing between important and irrelevant information. Neurooscillatory deficits have been observed in this ability in individuals diagnosed with schizophrenia.

The current study investigated whether mothers with schizotypy $(n=33)$ and their 6-month-old infants $(n=38)$ display reduced evoked-oscillatory activity. The mothers completed the Oxford-Liverpool Inventory of Feelings and Experiences - Short Form as an index of schizotypy dimensionality, which was used to categorise the participants into infants of control mothers and mothers with schizotypy. The paired-tone paradigm: two identical auditory tones (Stimulus 1 and Stimulus 2) played 500ms apart, were used to probe evoked oscillatory activity.

Data revealed that although the infants' evoked-oscillations displayed differences between Stimulus 1 and Stimulus 2 , there were no group differences between infants of mothers with schizotypy and of control mothers. Their mothers, however, displayed differences, with reduced power toward Stimulus 1 observed in the mothers with schizotypy between $13-30 \mathrm{~Hz}$. These findings are consistent that early sensory processes, such as sensory gating are impaired in schizophrenia-spectrum disorders.

\section{Funding statement}

Eleanor Smith is fully funded by the Leverhulme Trust Doctoral Scholarship Programme in Interdisciplinary Research on Infant Development.

\section{Ethics statements}

(Authors are required to state the ethical considerations of their study in the manuscript, including for cases where the study was exempt from ethical approval procedures)

Does the study presented in the manuscript involve human or animal subjects: Yes

Please provide the complete ethics statement for your manuscript. Note that the statement will be directly added to the manuscript file for peer-review, and should include the following information:

- Full name of the ethics committee that approved the study

- Consent procedure used for human participants or for animal owners

- Any additional considerations of the study in cases where vulnerable populations were involved, for example minors, persons with disabilities or endangered animal species 
As per the Frontiers authors guidelines, you are required to use the following format for statements involving human subjects: This study was carried out in accordance with the recommendations of [name of guidelines], [name of committee]. The protocol was approved by the [name of committee]. All subjects gave written informed consent in accordance with the Declaration of Helsinki.

For statements involving animal subjects, please use:

This study was carried out in accordance with the recommendations of 'name of guidelines, name of committee'. The protocol was approved by the 'name of committee'.

If the study was exempt from one or more of the above requirements, please provide a statement with the reason for the exemption(s).

Ensure that your statement is phrased in a complete way, with clear and concise sentences.

This study was carried out in accordance with the recommendations of Lancaster University Ethics Board and the North-West Lancaster Research Ethics Committee for the NHS with written informed consent from all subjects. All subjects gave written informed consent in accordance with the Declaration of Helsinki. The protocol was approved by the Lancaster University Ethics Board and the North-West - Lancaster Research Ethics Committee for the NHS. 


\section{The influence of Schizotypy on Event-Related Oscillations in Sensory}

\section{Gating during early Infant Development.}

Eleanor S. Smith ${ }^{1,2}$, Trevor J. Crawford ${ }^{1}, \&$ Vincent M. Reid ${ }^{1}$.

${ }^{1}$ Department of Psychology, Lancaster University, Bailrigg, Lancaster, United Kingdom, LA1 4YF.

${ }^{2}$ Department of Experimental Psychology, Downing Site, Downing Street, Cambridge, United Kingdom, CB2 3EB.

Corresponding author: Eleanor S. Smith: ess46@cam.ac.uk

There are no conflicts of interest to declare.

Funding: The present research was funded by the Leverhulme Trust Doctoral Scholarship Programme in Interdisciplinary Research on Infant Development. 


\begin{abstract}
Maternal personality is known to influence childhood risk factors for mental health. More specifically, maternal psychopathologies, such as those on the schizophrenia-spectrum have been associated with P50 sensory gating abilities. Schizotypy is a personality dimension within the general population elevated among schizophrenia-spectrum patients and their firstdegree relatives. Sensory gating is the pre-attentional habituation of responses distinguishing between important and irrelevant information. Neuro-oscillatory deficits have been observed in this ability in individuals diagnosed with schizophrenia.

The current study investigated whether mothers with schizotypy $(n=33)$ and their 6-month-old infants $(n=38)$ display reduced evoked-oscillatory activity. The mothers completed the Oxford-Liverpool Inventory of Feelings and Experiences - Short Form as an index of schizotypy dimensionality, which was used to categorise the participants into infants of control mothers and mothers with schizotypy. The paired-tone paradigm: two identical auditory tones (Stimulus 1 and Stimulus 2) played 500ms apart, were used to probe evoked oscillatory activity.

Data revealed that although the infants' evoked-oscillations displayed differences between Stimulus 1 and Stimulus 2, there were no group differences between infants of mothers with schizotypy and of control mothers. Their mothers, however, displayed differences, with reduced power toward Stimulus 1 observed in the mothers with schizotypy between $13-30 \mathrm{~Hz}$. These findings are consistent that early sensory processes, such as sensory gating are impaired in schizophrenia-spectrum disorders.
\end{abstract}

Keywords: EEG; Event-related Oscillations; Sensory Gating; Schizophrenia; Schizotypy; Infancy 


\section{Introduction}

Maternal personality is known to play an influential role in determining childhood risk factors for mental health. For example, specific maternal psychopathologies have been associated with P50 sensory gating abilities, illustrated through event-related potentials (Ross and Freedman, 2015). Atypical P50 sensory gating is an established biological trait of schizophrenia (Raine, 2006), which is also observed in individuals with schizotypal personality disorder (Cadenhead et al., 2000) and schizotypic personality traits, but who remain in the general population (Smith et al., 2019). These deficits are also observed in infants and children of parents with psychoses or severe anxiety disorders (Ross and Freedman, 2015). The presence of this deficit amongst all capacities of the schizophrenia-continuum supports sensory gating as a potential biomarker for the general risk of psychopathology. Despite the potential of this endophenotype to extend into infancy (Freedman et al., 2002; Smith et al., 2019) and to shape the development of personality into adulthood (Corr, 2010), the core neuropsychological and neuro-oscillatory dysfunctions of potential future psychopathologies that could be present during infancy or childhood, and may be related to the risk of developing psychosis is still unresolved (Debbané and BarrantesVidal, 2015). It is therefore of fundamental interest to explore spectral frequency analyses and auditory sensory gating within the non-clinical realm of the schizophrenia-spectrum and to explore whether these endophenotypic deficits are observed earlier in the developmental trajectory.

The inhibition of responses to irrelevant stimuli is an essential cognitive ability for humans in everyday life. The ability to 'gate out' irrelevant stimuli is known as sensory gating. This can be demonstrated as an attenuated neural response to the 
second identical stimulus in a paired-tone paradigm, which is considered an automatic inhibitory function (Freedman et al., 1987). In the auditory modality, the paired-tone paradigm: two tones presented in close succession, have been widely applied across the schizophrenia-spectrum (Patterson et al., 2008). The P50 auditory event-related potential (ERP) response to the second stimulus $(S 2)$ is typically reduced compared with that of the first stimulus (S1). This suppression, termed P50 gating, is thought to serve as a protective mechanism against flooding of the higher-order cortical centres with unnecessary information (Braff et al., 2007), with patients with schizophrenia and a proportion of their clinically unaffected relatives exhibiting reduced P50 ERP suppression. It is suggested that a sub-component of ERPs to paired-tones is a lowfrequency response occupying the $1-20 \mathrm{~Hz}$ range (Clementz and Blumenfeld, 2001). This range spans the beta band, associated with the detection of salient changes in sensory stimuli (Haenschel et al., 2000) thought to signify attentional engagement to task-relevant features of stimulus processing. This $1-20 \mathrm{~Hz}$ bandwidth has previously been employed in the paired-tone procedure to facilitate spectral power (Clementz and Blumenfeld, 2001; Johannesen et al., 2005) as putative indices of selective attention. As such, separating P50 ERPs into low-frequency response bands provides a broader assessment of auditory gating dysfunction (Johannesen et al., 2005).

Like P50 sensory gating, neuro-oscillatory deficits are also observed in the firstdegree relatives of schizophrenia patients, who are not on antipsychotic medication but exhibit schizotypic-personality traits (Hong et al., 2004). The shared experience of oscillatory sensory gating deficits raises the possibility that oscillatory abnormalities, in addition to event-related P50 gating, may present an endophenotype for schizophrenia-spectrum disorders (Gottesman and Gould, 2003). Thus, if atypical event-related oscillations are observed across the clinically diagnosable portion of the 
schizophrenia-spectrum (Gur et al., 2007; Seidman et al., 2006), and we know that P50 gating is observed among members of the general population who exhibit nonclinical schizotypic traits (Smith et al., 2019), then could these oscillatory abnormalities also be observed, to a lesser degree, in sub-clinical schizotypy, and more so, in their 6-month-old offspring? No research, as far as we are aware, has explored the oscillatory activity during auditory sensory gating among non-clinical mothers with schizotypy and their 6-month-old infants. However, whether deficits are observed among their 6-month-old infants is unknown as the literature is mixed in this instance, some suggesting that first-degree relatives display a similar deficit, but others suggesting that typical first-degree relatives of those diagnosed with schizophrenia display no difference in oscillatory gating compared to controls (Hong et al., 2008).

The underlying mechanisms involved in stimulus-evoked oscillations have been identified as being impaired among individuals diagnosed with schizophrenia, whom demonstrate reduced activity at low frequencies, including beta and gamma, to Stimulus 1 in a paired-tone paradigm, when compared to controls in fronto-central regions (Freedman et al., 1987; Johannesen et al., 2005). These gamma abnormalities have been related to early auditory processing (Hong et al., 2008) such as sensory gating. Poor gating may, in part, reflect attenuated neural activation in response to $S 1$ ('gating in' deficits) between 1-20Hz (Johannesen et al., 2005), which is hypothesised as a predisposition to the misperception of environmental stimuli, although, this is not observed in all patients (Clementz and Blumenfeld, 2001), suggesting a disrupted or inefficient formation of neural assemblies for registering sensory input. 
The auditory P50 ERP, which has been shown to be atypical among individuals who display schizotypic traits but who remain part of the general population (Smith et al., 2019), overlaps morphologically with P50 gating responses to $S 1$ in the evoked gamma frequency (approximately $40 \mathrm{~Hz}$ ) and in the low beta frequency range (approximately 16Hz, see Kisley and Cornwell, 2006), both of which have been negatively associated with the P50 gating response to $S 2$ (Hong et al., 2008). Beta $(\sim 13-29 \mathrm{~Hz})$ oscillations are associated with encoding and consolidating sensory information. The current literature suggests that gamma $(35-45 \mathrm{~Hz})$ and beta oscillations $(13-30 \mathrm{~Hz})$ may therefore contribute to auditory P50 ERPs (see Uhlhaas and Singer, 2010, for a review), although the precise mechanisms involved remain to be determined. Based on the proposed role(s) of activity in these frequencies for stimulus processing, it is possible that sensory flooding is associated with the inability to reduce beta and gamma power to reduce stimulus salience following repetitive stimulation.

With reference to the present research's aims, no research as far as we are aware has explored the oscillatory activity in response to auditory sensory gating among nonclinical schizotypy mothers and their 6-month-old offspring. With reductions in evoked gamma power present early in the course of schizophrenia-spectrum disorders, and possibly even prior to the onset of behavioural symptoms during the prodromal period, it may be possible to observe similar activation patterns in the subclinical portion of the schizophrenia-spectrum and amongst the infant first-degree relatives of these sub-clinical individuals. 
Reduced gamma power and synchrony deficits have been positively correlated with the negative symptomatology of schizophrenia (Lee et al., 2003). Hong et al., 2008, however, demonstrated how the presence of typical gamma and beta gating in relatives suggests that the underlying cognitive functions measured by the eventrelated oscillation gating responses may differ from those tapped by P50 gating. As such, the primary aim of the current research was to observe whether mothers who identify as exhibiting schizotypic traits demonstrate reduced evoked power in their neuro-oscillatory responses during a sensory gating paradigm (Experiment 1 ). The present study also aims to explore whether these oscillatory responses have similar manifestations in their 6-month-old offspring (Experiment 2), or whether no differences are observed among their first-degree relatives. We hypothesised that the mothers with schizotypy would illustrate reduced oscillatory power in the gamma and beta frequency ranges in response to $S 1$ and that, if their infants were to demonstrate a similar deficit, that they would present this atypicality to a lesser degree. 


\section{Method}

\section{Experiment 1: Adult Cohort}

Participants

Forty-three mothers ( $M$ age $=32.9$ years, $S D=3.6$ years $)$ participated in the research and were recruited from the Lancaster University Department of Infant and Child Development database. All participants were from a non-clinical population but completed the Oxford-Liverpool Inventory of Feelings and Experiences- Short Form (sO-LIFE; Mason, Linney, and Claridge, 2005), which is a four-scale questionnaire used to measure psychosis-proneness, principally schizotypy, in healthy individuals in the general population (Mason and Claridge, 2006). Thirty-three mothers were included in the final analysis. The final sample included twenty participants who identified as a schizotypic mother (SZTm) and the remaining thirteen participants were controls (CONm). Ten participants were excluded from the final sample due to: poor data quality $(n=1)$, and their sO-LIFE score not identifying with either control of schizotypic groups $(n=9)$. The mean of the sO-LIFE total score across the population was calculated (total $M=10.07$, total $S D=6.77$ ) and the $S Z T m$ condition was determined by the $M+.5 S D$ (sO-LIFE Scores $>13.46$ ) and the CONm condition by the $M-.5 S D$ (sO-LIFE Scores 6.68>0.0). This criterion was used as a result of its previous use in the schizotypy literature (for example, Park et al., 2015), thus if the participants sO-LIFE total score did not fall into one of the two categories then they were excluded from group analyses $(n=9)$. Ethical approval was obtained with the Lancaster University Ethics Board in accordance with the Declaration of Helsinki and the North West - Lancaster Research Ethics Committee for the NHS. All participants gave written informed consent for the study. 


\section{Experiment 2: Infant Cohort.}

Participants

One-hundred and one infants, aged 6-months ( $M=5.8$ months; $S D=9.23$ days; 54 male) participated in the study and were the biological offspring of the previously discussed maternal cohort. A further sixty-four were excluded from the final sample due to: poor data quality $(n=18)$, no auditory data collected due to the infants not sleeping ( $n=26)$, sO-LIFE score did not identify with either control or schizotypic groups $(n=10)$, or the participant did not produce over $20 \%$ good epochs $(n=10)$. Thirty-seven (20 male) infants were included in the final analysis (sO-LIFE Score total $M=8.15$, total $S D=6.26$ ). The final sample included fifteen participants identifying as an infant of a schizotypic mother (iSZTm; determined by the $M+.5 S D$ (sO-LIFE Scores $>11.28)$ ) and the remaining twenty-two participants were infants of control mothers (iCONm; determined by $M-.5 S D$ (sO-LIFE Scores 5.02>0.0)). All participants were from a non-clinical population. Ethical approval was obtained with the Lancaster University Ethics Board in accordance with the Declaration of Helsinki and the North West - Lancaster Research Ethics Committee for the NHS. All parents of infant participants gave written informed consent for the participation of their infant in the present study.

A 6-month-old infant population was chosen due to the developmental trajectories observed in the existing sensory gating literature (for example, Smith et al., 2019). We know from the literature that sensory gating can be observed from 2- (Hutchison et al., 2017) or 3-months of age (Hunter et al., 2015), although there are inconsistencies in developmental trajectories due to large age-gaps amongst published literature. In respect to the present attrition rate, for an EEG experiment with infants, this is a typical sample size for studies using these methods (e.g., Stets et al., 2012; 
Smith et al., 2019) or substantially greater than the sample size for studies on schizotypy during development (Hunter et al., 2015).

An independent samples t-test and Chi-squared analysis presented no significant differences between both schizotypic and control groups in both infant (Table 1) and maternal cohorts (Table 2). Despite no significant differences observed between the groups, it can be observed that there is a marginally greater incidence of mental health experience among control mothers.

Table 1. Table indicating similarities between the iSZTm and iCONm groups in their demographic information, as collected using a general information questionnaire.

\begin{tabular}{|c|c|c|c|c|}
\hline & $\begin{array}{c}\text { Infants of } \\
\text { Mothers with }\end{array}$ & Infants of Control & $p$ - & Chi- \\
& Schizotypy & $(n=22)$ & value & square \\
& $(n=15)$ & $M(S D)$ & & \\
\hline Infant Age (days) & $179.27(9.51)$ & $178.52(7.77)$ & .793 & \\
\hline Female & $n=6$ & $n=12$ & & .476 \\
\hline Male & $n=9$ & $n=11$ & & \\
\hline Mothers Mental Health & $1.60(.51)$ & $1.86(.35)$ & .070 & \\
Experiences & & & & \\
\hline Family History of Mental Health & $1.53(.52)$ & $1.59(.50)$ & .737 & \\
\hline Birth Complications & $2.00(1.00)$ & $1.57(.84)$ & .158 & \\
\hline
\end{tabular}


Table 2. Table indicating similarities between the SZTm and CONm groups in their demographic information, as collected using a general information questionnaire.

\begin{tabular}{|c|c|c|c|c|}
\hline & $\begin{array}{c}\text { Mothers with } \\
\text { Schizotypy } \\
(n=20)\end{array}$ & $\begin{array}{c}\text { Control Mothers } \\
(n=13)\end{array}$ & $\begin{array}{c}p \text { - } \\
\text { value }\end{array}$ & square \\
\hline Mothers Age (years) & $32.53(5.21)$ & $32.69(2.06)$ & .914 & \\
\hline Mental Health Experiences & $1.60(.51)$ & $1.86(.35)$ & .070 & \\
No Mental Health Experience & $n=8$ & $n=19$ & & .094 \\
Depression & $n=3$ & $n=2$ & & \\
Anxiety & $n=0$ & $n=1$ & & \\
Postnatal Depression & $n=2$ & $n=0$ & & \\
Depression/Anxiety & $n=2$ & $n=0$ & & \\
\hline Family History of Mental Health & $1.53(.52)$ & $1.59(.50)$ & .737 & \\
\hline
\end{tabular}

\section{Procedure.}

Prior to participation, the caregiver completed a series of questionnaires, which included the Oxford and Liverpool Inventory of Feelings and Experiences (sO-LIFE; Mason, Linney, and Claridge, 2005) as a measure of schizotypy dimensionality. The EEG cap was soaked in a warm water, sodium chloride solution and baby shampoo before fitting to the participant's head prior to the infant falling asleep or the mother being made comfortable in a darkened room. Once fitted and following confirmation that each electrode responded to electrical activity, the trial procedure began. The auditory stimuli was presented 80 -centimetres away, between $70-77 \mathrm{~dB}$ in serial presentation until the 15-minutes were complete, the infant woke or became restless. The infant was then left to complete their natural sleep period. Throughout the testing period the participant's status was video-recorded to index activity. The 
biological mothers of the infant cohort were invited back to participate in the same paradigm at a later date in a darkened room in a resting state.

The rationale for carrying out the infant portion of the paradigm while they were sleeping is based on research by Hunter et al. (2015), who explored sensory gating among sleeping children ( 4-years-old). Previous research had verified that like adults, REM sleep was a favourable state in which to measure P50 sensory gating in infants as movement artefacts are reduced (Kisley et al., 2003).

Materials and Stimuli

The materials, stimuli, and analysis utilised were the same for Experiment 1 and Experiment 2 with the exception of the scalp regions and time windows for analysis. All participants experienced a pair of single-sound stimuli that were based on Park, Lim, Kirk, and Waldie (2015). See Figure 1 for a schematic of the paired-tone paradigm. Two identical tones (Stimulus 1: S1, and Stimulus 2: S2) were presented with a $500 \mathrm{~ms}$ inter-tone interval every 10 -seconds for 15 -minutes or until the infant woke. The paired tones were presented between $70-77 \mathrm{~dB}$ and had a tonal quality of $1000 \mathrm{~Hz}$.

The mothers contributed on average $88.2 \%$ artefact-free trials $(M=49.48$ trials, $S D=5.35$ trials, range: $42-61$ trials) for $S 1$ and on average $88.3 \%$ artefact-free trials ( $M=49.58$ trials, $S D=5.12$ trials, range: $41-62$ trials) for $S 2$. There were no significant differences in trial numbers between $S 1$ and $S 2(t(32)=-.21, p=.84)$. The infants experienced a range of 63-140 paired-stimuli repetitions, dependent on length of sleep, and contributed an average of $51.62 \%$ artefact-free trials (range: $23-90.7 \%$ ) for 
$S 1$, and on average $52.04 \%$ artefact-free trials (range: $24.5-94 \%$ ) for $S 2$. There were no significant differences in trial numbers between $S 1$ and $S 2(t(44)=.98, p=.33)$

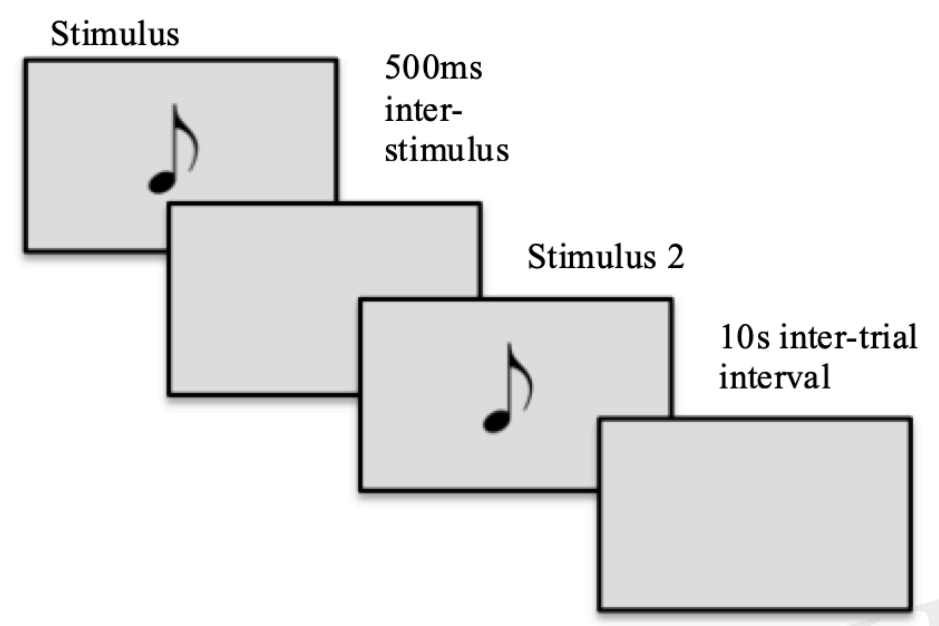

Figure 1. A graphical representation of the paired-tone paradigm. The tones were presented between $70-77 \mathrm{~dB}$ with a tonal quality of $1000 \mathrm{~Hz}$.

All electrophysiological signals were recorded using Electrical Geodesics Inc. amplifiers (input impedance $=80 \mathrm{~K} \Omega$; sampling rate $=500 \mathrm{~Hz}$ ) and event-related oscillations were measured using an EGI Hydrocel GSN-128 electrode 1.0 net and pre-processed using Netstation 4.5.4. All EEG signal processing and analyses were performed blind to group membership. The data was $0.5-65 \mathrm{~Hz}$ bandpass filtered and segmented to create epochs from $400 \mathrm{~ms}$ before to $1200 \mathrm{~ms}$ after stimulus-onset for each trial. For the elimination of electrical artefacts caused by eye and body movements, data was rejected offline by the visual editing of trial-by-trial data. This was carried out in conjunction with an artefact detection toolset in Netstation, which highlighted whether a channel was 'bad' for more than $80 \%$ of the recording, which was determined by a threshold of $200 \mu \mathrm{V}$ to remove outlier values resulting from artefacts, or if they contained more than 12 bad channels in a trial. Participants required a minimum of $20 \%$ good trials for each stimulus to be included in further analyses. 
Based on previous literature (Smith et al., 2010; Popov et al., 2011) and visual inspection of the grand and individual means, we selected the scalp area, time window and frequency band for the maternal and infant cohorts. For the maternal cohort, Gamma and Beta induced frequencies were measured over the left-frontal (the average of channels $12,18,19,20,22,23,24,26,27)$, and right-frontal (the average of channels $3,4,5,9,10,11,118,123,124)$ regions, in the $50-250 \mathrm{~ms}$ time window (Figure 2). Beta activity was analysed between $13-20 \mathrm{~Hz}$ and gamma between 35 50Hz. For the infant cohort, Gamma and Beta induced frequencies were measured over the left-temporal (the average of channels $47,51,52$ ), right-temporal (the average of channels 115,116 ), and left-frontal (the average of channels $12,20,24$ ) regions, in the $100-325 \mathrm{~ms}$ time window (Figure 2 ). Beta activity was analysed between $10-20 \mathrm{~Hz}$ and gamma between $30-50 \mathrm{~Hz}$.

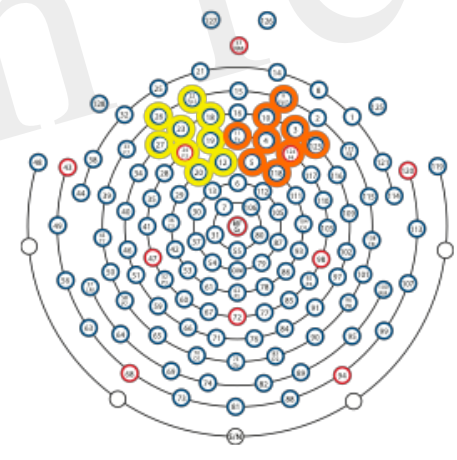

Maternal Regions of Interest

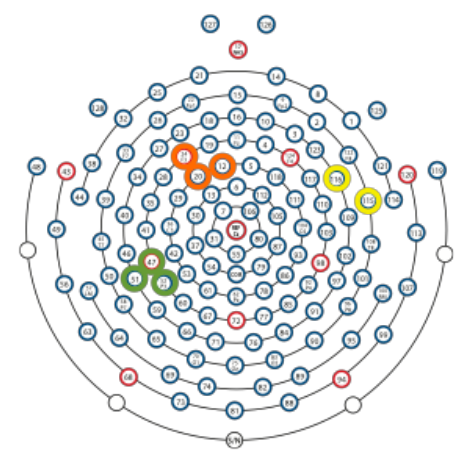

Infant Regions of Interest

Figure 2. Maternal and Infant regions of interest, which were averaged across the $50-250 \mathrm{~ms}$ and $100-325 \mathrm{~ms}$ time windows, respectively.

The artefact-free segments were subjected to time-frequency analysis to examine stimulus-induced oscillatory responses. The epochs were imported into Matlab ${ }^{\circledR}$ using the free toolbox EEGLAB (v. 9.0.5.6b) and re-referenced to the average reference. Using a custom-made scripts collection named 'WTools" (see Parise, Csibra, and Becchio, 2013, for reference) we computed complex Mortlet wavelets for the frequencies $10-65 \mathrm{~Hz}$ with $1 \mathrm{~Hz}$ resolution. Total-induced oscillations were 
calculated, performing a continuous wavelet transformation of all epochs by means of convolution with each wavelet and taking the absolute value (i.e., the amplitude, not the power) of the results (Csibra et al., 2000). Transformed epochs were then averaged for each condition separately. To remove the distortion introduced by the convolution, we edited out $200 \mathrm{~ms}$ at the edges of the epochs, resulting in $1200 \mathrm{~ms}$ long segments, including $200 \mathrm{~ms}$ before and $1000 \mathrm{~ms}$ after stimulus onset. We used the average amplitude of the $200 \mathrm{~ms}$ pre-stimulus window as baseline, subtracting it from the whole epoch at each frequency (Parise et al., 2013).

Questionnaires.

Schizotypy

The Oxford-Inventory of Feelings and Experiences- Short Form (sO-LIFE; Mason et al., 2005) assessed schizotypy dimensionality and divided the participant cohort into iSZTm/iCONm and SZTm/CONm. The sO-LIFE was chosen as the present measure of schizotypy dimensionality due to its fully dimensional approach, proposing that symptoms occurring in the schizophrenia-spectrum also occur in the typical population. The reliability of the sO-LIFE, estimated with a Cronbachs alpha of 0.79 was observed in the present cohort; illustrating a similar reliability estimate to prior literature (for example, 0.78; Fonseca-Pedrero et al., 2014).

\section{Personality Assessment.}

A shortened version of the EPQ-R personality questionnaire (Eysenck and Eysenck, 1992) was used as a measure of neuroticism in the mothers. There is a substantial overlap between schizotypy and neuroticism in typical participants (Ettinger et al., 2005) with sizeable correlations observed, and higher levels of neuroticism in individuals diagnosed with schizophrenia (Camisa et al., 2005). The shortened version of the EPQ-R included 12 self-reported 'yes/no' items, with an affirmative answer 
contributing one point. The present study used only the neuroticism subscale of the Eysenck Personality Questionnaire-Revised (EPQ-R; Eysenck and Eysenck, 1992), which has good internal consistency (alpha $=.85)$ and strong concurrent validity with related constructs (Stewart et al., 2005).

Additional Demographic Variables.

A general assessment questionnaire was used to gain an overall assessment of smoking habits, hearing deficits, birth complications, and whether they, or their family have experienced mental illness. See Table 1 for the outcome of this questionnaire.

Rationale for Analysis.

As outlined in the introduction, responses to $S 1$ in the beta frequency range have been negatively correlated with the P50 suppression response to $S 2$ in the paired-tone paradigm (Hong et al., 2008). For this reason, the statistical analysis will predominantly focus on oscillatory responses to S1. Firstly, a 2 (group: SZTm or $\mathrm{CONm}$ ) x 2 (paired-tone: $S 1$ or $S 2)$ x 2 (Region of Interest: right-frontal or leftfrontal) repeated-measures ANOVA will be carried out to explore the maternal beta oscillatory power response to $S 1$ and $S 2$. If a significant group difference is observed then we will explore group differences specifically in SI. If a group*region of interest interaction is observed then we will explore whether these effects are driven by the right- or left-frontal regions. A similar strategy will be employed with the infant data. Due to the dimensional nature of the schizophrenia-spectrum, in preference to a binary, between groups analysis, a more robust statistical approach explores the correlational relationships. Therefore, Pearson correlations will be used to explore the dimensional nature of sensory gating abilities within the range of sO-LIFE scores. For 
the present research, a result that yields a $p$-value of less than $0.05(p<0.05)$ have been labelled as significant.

Results

\section{Experiment 1: Maternal Cohort}

A 2 (group: SZTm or CONm) x 2 (paired-tone: $S 1$ or $S 2$ ) x 2 (Region of Interest: right-frontal or left-frontal) repeated-measures ANOVA explored the maternal beta oscillatory power response to $S 1$ and $S 2$. This illustrated how a significant main effect difference could be observed between $S 1$ and $S 2\left(\mathrm{~F}(1,31)=16.80, \mathrm{p}<.001, \eta_{\mathrm{p}}{ }^{2}=.35\right)$. A significant between-subjects effect $\left(F(1,31)=8.21, p=.007, \eta_{p}^{2}=.21\right)$ was also observed. The region of interest*group interaction was also found to be significant $\left(\mathrm{F}(1,31)=6.57, \mathrm{p}=.02, \eta_{\mathrm{p}}{ }^{2}=.18\right)$. No further comparisons were found to be significant. To explore these group differences further, a 2 (paired-tone: $S 1$ or $S 2$ ) x2 (group: SZTm or CONm) repeated-measures ANOVA corrected for multiple comparisons was carried out and illustrated a significant difference between the oscillatory activity towards the paired-tones $\left(\mathrm{F}(1,31)=18.38, \mathrm{p}<.001, \eta_{\mathrm{p}}{ }^{2}=.37\right)$, and a significant group effect $\left(F(1,31)=16.11, p<.001, \eta_{p}^{2}=.34\right)$. When exploring the group*paired-tone pairwise comparisons, both the SZTm and CONm demonstrate significant differences between their activity towards $S 1(M=.18, S D=.03)$ and $S 2(M=.07, S D=.03)$, but overall, the SZTm illustrated less beta activity in response to $\mathrm{S} 1(M=.09, S D=.02)$ and S2 $(M=-.02, S D=.02)$, replicating previous literature (Kisley and Cornwell, 2006; Hong et al., 2008). To explore these differences further, a paired-samples t-test illustrated a significant result in the right region $(t(32)=2.45, \mathrm{p}=.02,95 \%$ CI $[.01$, $.08]$ ); suggesting more negativity in $S 2$ as observed in the mother cohort (Figure 3). 


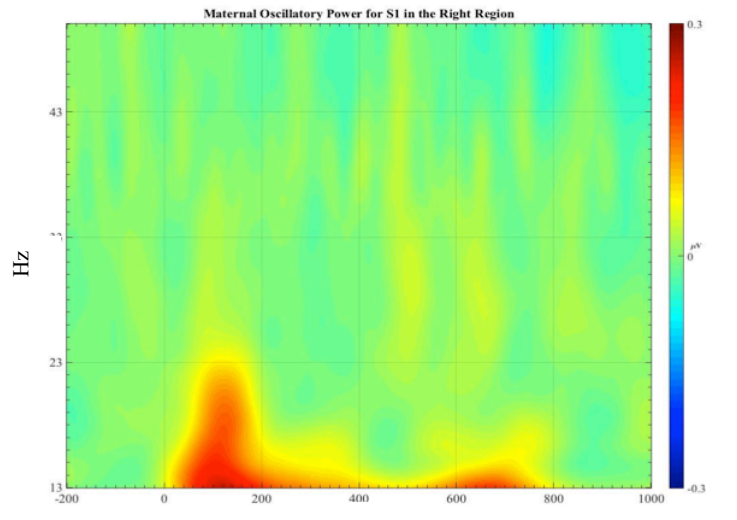

Ms

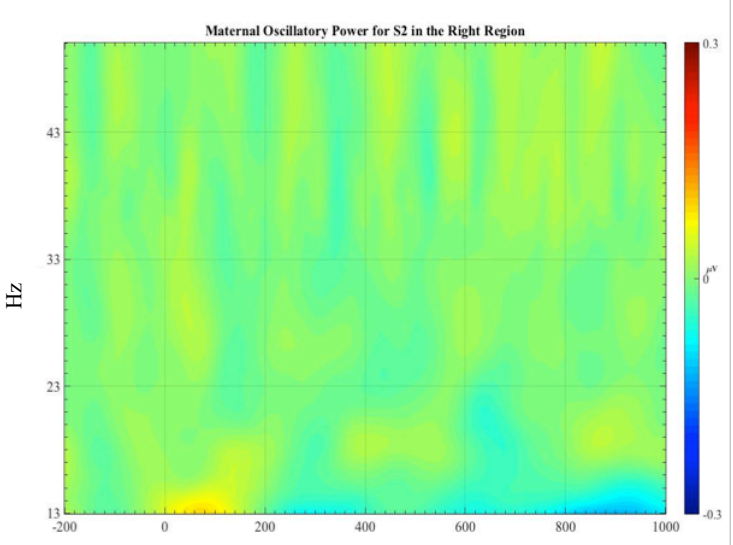

Ms

Figure 3. Topographical Plot of the $S 1$ and $S 2$ stimuli responses across the entire maternal cohort. Note how the power related to $S 1$ is greater in comparison to that of $S 2$.

To explore the groups oscillatory responses to $S 1$, a one-way ANOVA was utilised and demonstrated a significant between-groups effect in the right region $(\mathrm{F}(1,32)=7.58, \mathrm{p}=.01)$. When exploring the descriptive statistics in the right-frontal region, the CONm illustrate a greater response to $S 1(M=.18, S D=.12)$ in comparison to $\operatorname{SZTm}(M=.09, S D=.09)$. This supports the hypothesis that mothers with schizotypy show decreased activity in $S 1$ compared to control mothers between $13-20 \mathrm{~Hz}$ (beta; Figure 4). The present research hypothesised that the mothers with schizotypy would illustrate reduced oscillatory power in response to $S 1$; this hypothesis was supported by the data reported here. 

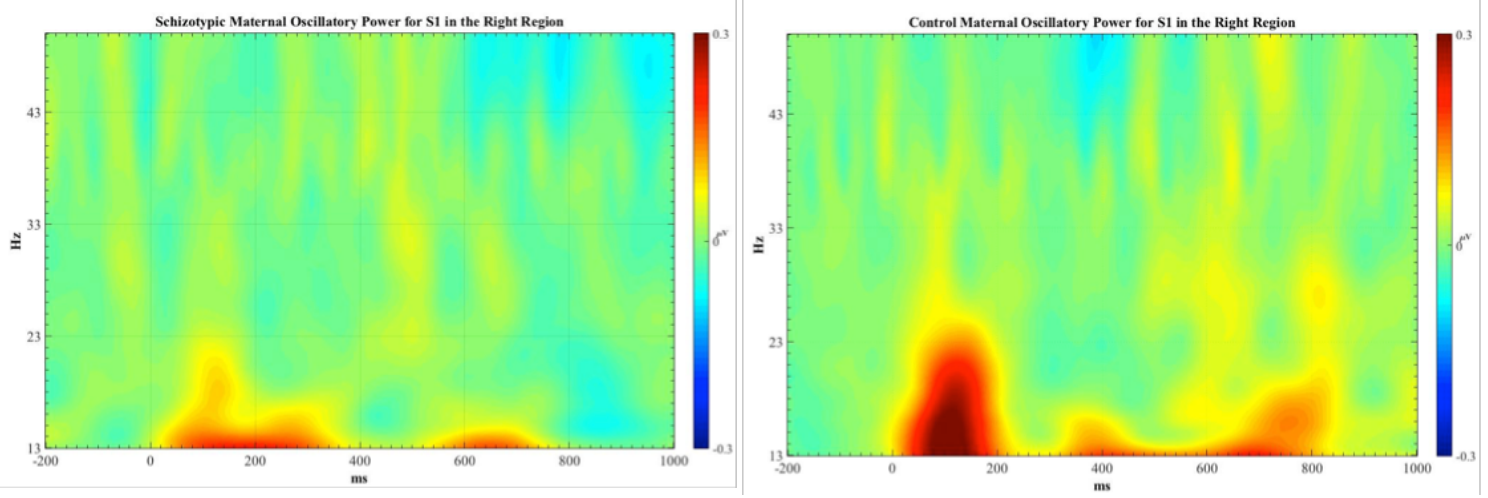

Figure 4. Topographical Plot of the $S 1$ stimuli of SZTm and CONm in the right region between $13-20 \mathrm{~Hz}$. Note how the CONm demonstrate greater oscillatory power when compared to their SZTm counterparts whom demonstrate a similar pattern of activation but to a lesser degree.

Pearson correlations between the maternal sO-LIFE total and dimension scores and the maternal oscillatory power exhibited in response to $S 1$ were carried out. Significant negative relationships were observed between the responses to $S 1$ in the right-frontal region and the sO-LIFE total $(n=32, r=-.425, p=.02)$, sO-LIFE Unusual Experiences dimension $(n=32, r=-.519, p=002)$, and the sO-LIFE Cognitive Disorganisation dimension $(n=32, r=-.385, p=.03)$. These relationships suggest that a greater sO-LIFE score, which is indicative of schizotypy dimensionality, the lower the oscillatory power exhibited towards $S 1$, supporting prior literature. No significant correlations were observed for the Introvertive Anhedonia or Impulsive Nonconformity dimensions. 

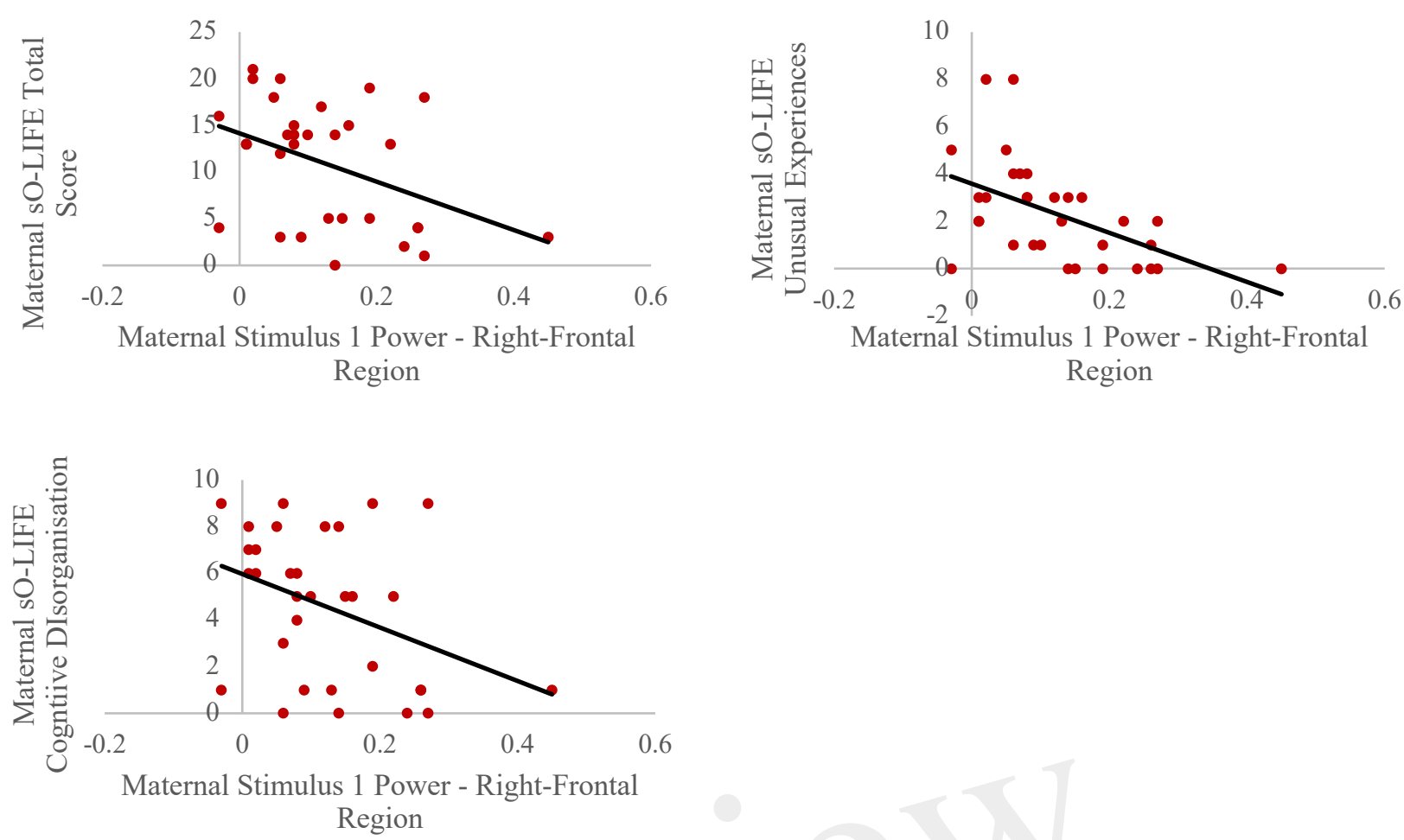

Figure 5. Pearson correlational analyses between the maternal sO-LIFE total and dimension scores and the maternal oscillatory power exhibited in response to $S 1$ in the right-frontal regions.

\section{Experiment 2: Infant Cohort}

Results

A 3 (region of interest: left-frontal, left-temporal, right-temporal) x 2 (paired-tone: $S 1$ or $S 2$ ) x 2 (group: iSZTm or iCONm) repeated-measures ANOVA, with Bonferroni Post-Hoc tests for multiple comparisons, illustrated a significant difference between beta activity towards $S 1$ and $S 2\left(\mathrm{~F}(1,36)=6.89, \mathrm{p}<.01, \eta_{\mathrm{p}}{ }^{2}=.16\right)$, and a trend towards a paired-tone by region of interest interaction $\left(F(2,72)=2.85, \mathrm{p}=.06, \eta_{\mathrm{p}}^{2}=.07\right)$. No further comparisons were found to be significant. To further explore the differences between activity in the different regions of interest, a paired-samples t-test suggested a significant difference between oscillatory power toward $S 1$ and $S 2(\mathrm{t}(37)=2.82$, $\mathrm{p}=.008$ ). When exploring the group by paired-tone interaction for pairwise 
comparisons, it became apparent that the iSZTm did not display a significant difference between $S 1$ or $S 2$ ( $\mathrm{p}=.09)$, but the iCONm did show a significant difference between the two stimuli ( $p=.04)$; this does not, however, drive a significant group effect. A paired-samples t-test was undertaken to illustrate a significant difference between the frequencies observed in the right-temporal region $(\mathrm{t}(37)=2.82, \mathrm{p}=.008$, 95\% CI [.02,.13]); illustrating decreased activity in $S 2$ compared to $S 1$ (Figure 6). No further significant effects were observed. We hypothesised that if the infants were to demonstrate a similar deficit to their biological mothers, then they too would exhibit reduced oscillatory power in the gamma and beta frequency ranges in response to $S 1$. The current data, however, did not show these deficits in the infant cohort, supporting the results of Hong et al. (2008) in that typical first-degree relatives of those diagnosed with schizophrenia display no difference in oscillatory gating compared to controls.

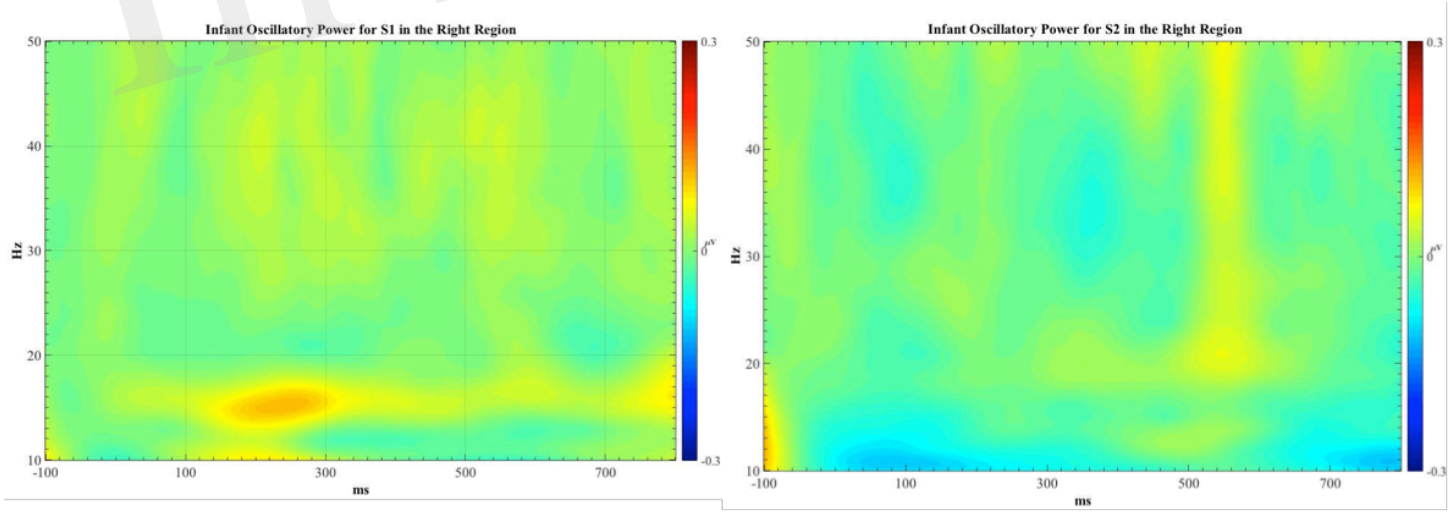

Figure 6. Topographical Plot of $S 1$ and $S 2$ across the entire infant cohort. Note how the power related to $\mathrm{S} 1$ is greater in comparison to that of $S 2$. A greater power can be observed between $10-20 \mathrm{~Hz}$ across the time-window of $S 1$.

Pearson correlations between the maternal sO-LIFE total and dimension scores and the infant oscillatory power exhibited in response to $S 1$ were carried out. 
No significant relationships were observed between these dimensions and the infant oscillatory power in the left-temporal, right-temporal, or left-frontal regions.

\section{General Discussion}

The present research aimed to observe whether mothers with schizotypy demonstrate reduced evoked oscillations during sensory gating, and whether these deficits are replicated in their 6-month-old offspring. Results demonstrated how mothers with schizotypy displayed reduced beta activity towards $S 1$, replicating previous literature (Johannesen et al., 2005; Brenner et al., 2009; Hall et al., 2011). In contrast, the infants showed no significant differences between groups; suggesting that having a mother with schizotypy did not influence the infants' oscillations in relation to sensory gating. These findings support our maternal hypotheses stating whether these deficits are observed to a lesser degree in sub-clinical schizotypy, and illustrate parallels with Hong et al. (2008) in that the first-degree relatives of those on the schizophrenia-spectrum display no difference in their event-related oscillation gating responses compared to controls.

It should be articulated that schizotypy, for the purpose of the current research, was defined using the sO-LIFE, with mothers classed as schizotypic if their sO-LIFE score was half a standard deviation above the mean; weighing in favour of a fullydimensional approach and replicating the approach taken by Park et al. (2015). However, this approach could limit our understanding of schizotypy as a construct with separable and well-identified components (Kwapil, Barrantes-Vidal, and Silvia, 2008). To account for this, the present research included correlational analyses between the sO-LIFE total and dimension scores in comparison to the oscillatory 
power toward $S 1$. This found that mothers with greater sO-LIFE scores: an indicator of schizotypy dimensionality, illustrated lower oscillatory power towards $S 1$. This was not however, observed in their infants.

There is growing research into schizotypy and its involvement in the schizophreniaspectrum. Exploration into the oscillatory frequencies of first-degree relatives of individuals on this spectrum, in the form of their 6-month-old offspring, is an integral step forward in the literature, which holds the necessary ingredients to bring a developmental psychopathology account to psychotic disorders. The current research is the first time that this has been explored with evoked-oscillations, to our knowledge, and for this reason, the methodology was based on Park et al. (2015) and Ross and Freedman (2015).

Neural oscillatory activity in the beta range is thought to reflect the different aspects of early sensory information processing, with hypotheses that beta oscillations may contribute to sensory gating (Hong et al., 2004). The presence of typical gamma and beta gating in first-degree relatives (Hong et al., 2008) suggests that the underlying cognitive functions measured by the oscillatory gating responses may differ from those tapped by P50 suppression. The present research supports the notion that those individuals who display traits from the schizophrenia-spectrum also display differences in oscillatory function when contrasted with controls, however, the firstdegree relatives of these individuals do not present the same deficit.

Psychiatric disorders, such as schizophrenia, may be thought of as a 'shift' in the continuous distribution of neurodevelopmental traits towards greater impairment, whilst maintaining overlap with the population distribution (Hengartner and 
Lehmann, 2017). Establishing brain-behaviour links in both clinically significant behaviours and those of typical development is an important step in further understanding the continuities and discontinuities that exist between typical and pathological behaviour. Despite some literature finding low reliability (Luck et al., 2011), meta-analysis supports the relationship between sensory gating and schizophrenia (Patterson et al., 2008) and the method for measuring sensory gating in infants has been established (Kisley et al., 2003). The successful adaptation of tasks for use in early infancy therefore increases our understanding of the developmental timeline of these disorders and will perhaps allow for the development of novel prevention strategies.

Strength of the present research is in the multiple electrode sites utilised for analysis when contrasted to prior research, which used low-density montages or singular electrodes (for example, CZ, Hong et al., 2004). The present research also highlights the complexity of recording electrophysiological activity during sleep where infants produce unpredictable movements; increasing artefacts and attrition rates for inclusion in the final analyses. A limitation of the present study is the lack of previous literature observing sensory gating time-frequency oscillations with infants. This is the first time this has been demonstrated, to our knowledge. For this reason, there is little literature on which to base our predictions and have a priori topographical hypotheses; all topographical predictions and outcomes must therefore be made with caution.

The present research has illustrated how mothers with sub-clinical schizotypy who are part of the general population display reduced beta activity towards Stimulus 1 in a 
paired-tone paradigm; which parallels previous literature exploring this same mechanisms among other groups located on the schizophrenia-spectrum. This shared deficit in oscillatory sensory gating supports the potential contribution of oscillatory abnormalities of this kind as an endophenotype for the schizophrenia-spectrum, although further exploration and replication is needed. In contrast to the maternal cohort, the infants demonstrated no group differences, highlighting that having a mother with schizotypy did not influence their oscillatory sensory gating abilities at 6months-old.

Acknowledgements

This work is supported by the Leverhulme Trust Doctoral Scholarship Programme in Interdisciplinary Research on Infant Development. The authors wish to acknowledge Dr Eugenio Parise who assisted in the analysis of the data through the use of his MatLab custom-made scripts collection named 'WTools'. The authors also wish to acknowledge the involvement and support of the families who participated in the present research. 


\section{References}

Braff, D.L., Freedman, R., Schork, N.J., \& Gottesman, I.I. (2007). Deconstructing schizophrenia: an overview of the use of endophenotypes in order to understand a complex disorder.

Schizophrenia Bulletin, 33, 21-32. https://doi.org/10.1093/schbul/sb1049

Brenner, C.A., Kieffaber, P.D., Clementz, B.A., Johanessen, J. K., Shekhar, A., O`Donnell, B.F., \& Hetrick, W.P. (2009). Event-related potential abnormalities in schizophrenia: a failure to "gate in" salient information? Schizophrenia Research, 113, 332-338.

https://doi.org/10.1016/j.schres.2009.06.012

Cadenhead, K.S., Light, G.A., Geyer, M.A., \& Braff, D.L. (2000). Sensory gating deficits assessed by the P50 event-related potential in subjects with schizotypal personality disorder. The American Journal of Psychiatry, 157(1), 55-59. https://doi.org/10.1176/ajp.157.1.55

Camisa, K.M., Bockbrader, M.A., Lysaker, P., Rae, L.L., Brenner, C.A., \& O’Donnell, B.F. (2005). Personality traits in schizophrenia and related personality disorders. Psychiatry Research, 133(1), 23-33. https://doi.org/10.1016/j.psychres.2004.09.002

Clementz, B.A., \& Blumenfeld, L.D. (2001). Multichannels electroencephalographic assessment of auditory evoked response suppression in schizophrenia. Experimental Brain Research. 139, 377390. https://doi.org/10.1007/s002210100744

Corr, P. (2010). The psychoticism-psychopathy continuum: A neuropsychological model of core deficits. Personality and Individual Differences. 48(6), 695-703. https://doi.org/10.1016/j.paid.2009.12.023

Csibra, G., Davis, G., Spratling, M.W., \& Johnson, M.H. (2000). Gamma oscillations and object processing in the infant brain. Science, 290, 1582-1585. http://dx.doi.org/10.1126/science.290.5496.1582

Debbané, M., \& Barrantes-Vidal, N. (2015). Schizotypy from a developmental perspective. Schizophrenia Bulletin, 41(2), 386-95. https://doi.org/10.1093/schbul/sbu175

Ettinger, U., Kumari, V., Crawford, T.J., Flak, V., Sharma, T., Davis, R.E., \& Corr, P.J. (2005). Saccadic eye movements, schizotypy, and the role of neuroticism. Biological Psychology, 68, 61-78. https://doi.org/10.1016/j.biopsycho.2004.03.014 
Eysenck, H. J., \& Eysenck, S.B.G. (1992). Manual for the Eysenck Personality Questionnaire-

Revised. San Diego, CA: Educational and Industrial Testing Service.

Fonseca-Pedrero, E., Fumero, A., Paino, M., De Miguel, A., Ortuño-Sierra, J., Lemos-Giráldez, S., \& Muñiz, J. (2014). Schizotypal Personality Questionnaire: New sources of validity evidence in college students. Psychiatry Research, 219(1), 214-220.

https://doi.org/10.1016/j.psychres.2014.04.054

Freedman, R., Adler, L.E., Gerhardt, G.A., Waldo, M., Baker, N., Rose, G.M., Drebing, C., Nagamoto, H., Bickford-Wimer, P., \& Franks, R. (1987). Neurobiological studies of sensory gating in schizophrenia. Schizophrenia Bulletin, 13, 669-678.

https://doi.org/10.1093/schbul/13.4.669

Freedman, R., Adler, L.E., Olincy, A., Waldo, M.C., Ross, R.G., Stevens, K.E., \& Leonard, S. (2002). Input dysfunction, schizotypy, and genetic models of schizophrenia. Schizophrenia Research, 54, 25-32. https://doi.org/10.1016/S0920-9964(01)00348-6

Gottesman, I.I., \& Gould, T. D. (2003). The endophenotype concept in psychiatry: etymology and strategic intentions. American Journal of Psychiatry, 160, 636-45. https://doi.org/10.1176/appi.ajp.160.4.636

Gur, R.E., Nimgaonkar, V.L., Almasy, L., Calkins, M.E., Ragland, J.D., Pogue-Geile, M.F., Kanes, S., Blangero, J., \& Gur, R.C. (2007b). Neurocognitive endophenotypes in a multiplex multigenerational family study of schizophrenia. American Journal of Psychiatry, 164, 813819.

Haenschel, C., Baldeweg, T., Croft, R. J., Whittington, M, \& Gruzelier, J. (2000). Gamma and beta frequency oscillations in response to novel auditory stimuli: a comparison of human electroencephalogram (EEG) data with in vitro models. Proceedings of the National Academy of Sciences of the United States of America. 97, 7645-7650.

https://doi.org/10.1073/pnas.120162397

Hall, M.H., Taylor, G., Salisbury, D.F., \& Levy, D.L. (2011). Sensory gating event-related potentials and oscillations in schizophrenia patients and their unaffected relatives. Schizophrenia Bulletin, 37, 1187-1199. https://doi.org/10.1093/schbul/sbq027 
Hengartner, M.P., \& Lehmann, S.N. (2017). Why psychiatric research must abandon traditional diagnostic classification and adopt a fully dimensional scope: Two solutions to a persistent problem. Frontiers in Psychiatry, 8, 101. https://doi.org/10.3389/fpsyt.2017.00101

Hong, L., Buchanan, R., Thaker, G., Shepard, P., \& Summerfelt, A. (2008). Beta ( 16 Hz) frequency neural oscillations mediate auditory sensory gating in humans. Psychophysiology, 45(2), 197204. https://doi.org/10.1111/j.1469-8986.2007.00624.x

Hong, L.E., Summerfelt, A., McMahon, R., Adami, H., Francis, G., Elliott, A., Buchanan, R.W., \& Thaker, G. K. (2004). Evoked gamma band synchronization and the liability for schizophrenia. Schizophrenia Research, 70, 293-302. https://doi.org/10.1016/j.schres.2003.12.011

Hunter, S.K., Gillow, S.J., \& Ross, R.G. (2015). Stability of P50 auditory sensory gating during sleep from infancy to 4 years of age. Brain and Cognition, 94, 4-9. https://doi.org/10.1016/j.bandc.2014.12.004

Hutchison, A., Hunter, S., Wagner, B., Calvin, E., Zerbe, G., \& Ross, R. (2017). Diminished Infant P50 Sensory Gating Predicts Increased 40-Month-Old Attention, Anxiety/Depression, and Externalizing Symptoms. Journal of Attention Disorders, 21(3), 209-218. https://doi.org/10.1177/1087054713488824

Johannesen, J.K, Kieffaber, P.D., O’Donnell, B.F., Shekhar, A., Evans, J.D., \& Hetrick, W.P. (2005). Contributions of subtype and spectral frequency analyses to the study of P50 ERP amplitude and suppression in schizophrenia. Schizophrenia Research, 78, 269-284. https://doi.org/10.1016/j.schres.2005.05.022

Kisley, M.A., \& Cornwell, Z.M. (2006). Gamma and beta neural activity evoked during a sensory gating paradigm: Effects of auditory, somatosensory and cross-modal stimulation. Clinical Neurophysiology, 117, 2549-2563. https://doi.org/10.1016/j.clinph.2006.08.003

Kisley, M.A., Polk, S.D., Ross, R.G., Levisohn, P.M., \& \& Freedman, R. (2003). Early postnatal development of sensory gating. NeuroReport, 14, 693-697. 
Kisley, M. A., Polk, S. D., Ross, R. G., Levisohn, P. M., \& Freedman, R. (2003). Early postnatal development of sensory gating. Neuroreport, 14, 693-697.

Kwapil, T.R., Barrantes-Vidal, N., \& Silvia, P.J. (2008). The dimensional structure of the Wisconsin Schizotypy Scales: factor identification and construct validity. Schizophrenia Bulletin, 34, 444-457. https://doi.org/10.1093/schbul/sbm098

Lee, K.H., Williams, L.M., Haig, A., \& Gordon, E. (2003). "Gamma (40 Hz) phase synchronicity” and symptom dimensions in schizophrenia. Cognitive Neuropsychiatry, 8, 57-71. https://doi.org/10.1080/713752240

Luck, S.J., Mathalon, D.H., O’Donnell, B.F., Hämäläinen, M.S., Spencer, K.M., Javitt, D.C., \& Uhlhaas, P.J. (2011). A roadmap for the development and validation of event-related potential biomarkers in schizophrenia research. Biological Psychiatry, 70, 28-34. https://doi.org/10.1016/j.biopsych.2010.09.021

Mason, O., \& Claridge, G. (2006). The Oxford-Liverpool Inventory of Feelings and Experiences (OLIFE): Further description and extended norms. Schizophrenia Research, 82(2-3), 203-211.

Mason, O., Linney, Y., \& Claridge, G. (2005). Short scales for measuring schizotypy. Schizophrenia Research, 78(2), 293-296. https://doi.org/10.1016/j.schres.2005.06.02

Parise, E., Csibra, G., \& Becchio, C. (2013). Neural Responses to Multimodal Ostensive Signals in 5Month-Old Infants. PLoS ONE, 8(8), E72360. https://doi.org/10.1371/journal.pone.0072360

Park, H.R., Lim, V.K., Kirk, I.J., \& Waldie, K.E. (2015). P50 sensory gating deficits in schizotypy. Personality and Individual Differences, 82, 142-147. https://doi.org/10.1016/j.paid.2015.03.025

Patterson, J.V., Hetrick, W.P., Boutross, N.N., Jin, Y., Sandman, C., Stern, H., Potkin, S., \& Bunney, S.E. Jr. (2008). P50 sensory gating ratios in schizophrenics and controls: a review and data analysis. Psychiatry Research, 158, 226-247. https://doi.org/10.1016/j.psychres.2007.02.009 Popov, T., Jordanov, T., Rockstroh, B., Elbert, T., Merzenich, M.M., \& Miller, G.A. (2011). Specific Cognitive Training Normalizes Auditory Sensory Gating in Schizophrenia: A Randomized Trial. Biological Psychology, 69(5), 465-471. https://doi.org/10.1016/j.biopsych.2010.09.028 
Raine, A. (2006). Schizotypal personality: neurodevelopmental and psychosocial trajectories. Annual Reviews of Clinical Psychology, 2, 291-326.

https://doi.org/10.1146/annurev.clinpsy.2.02305.095318

Ross, R., \& Freedman, R. (2015). Endophenotypes in Schizophrenia for the Perinatal Period: Criteria for Validation. Schizophrenia Bulletin, 41(4), 824-834. https://doi.org/10.1093/schbul/sbv054 Seidman, L.J., Giuliano, A.J., Smith, C.W., Stone, W.S., Glatt, S.J., Meyer, E., Faraone, SV, Tsuang, M.T., \& Cornblatt, B. (2006). Neuropsychological functioning in adolescents and young adults at genetic risk for schizophrenia and affective psychoses: results from the Harvard and Hillside Adolescent High Risk Studies. Schizophrenia Bulletin, 32, 507-24. https://doi.org/10.1093/schbul/sbj078

Smith, A.K., Edgar, J.C., Huang, M., Lu, B.Y., Thoma, R.J., Hanlon, F.M., McHaffie, G., Jones, A.P., Paz, R.D., Miller, G.A., \& Cañive, J.M. (2010). Cognitive abilities and 50-and 100-msec paired-click processes in schizophrenia. American Journal of Psychiatry, 167(10), 1264-1275. https://doi.org/10.1176/appi.ajp.2010.09071059

Smith, E.S., Crawford, T. J., Thomas, M., \& Reid, V. M. (2019). Is schizotypic maternal personality linked to sensory gating abilities during infancy? Experimental Brain Research, 239, 18691879. doi: https://doi.org/10.1007/s00221-019-05554-7

Stets, M, Stahl, D., \& Reid, V.M. (2012). A meta-analysis investigating factors underlying attrition rates in infant ERP studies. Developmental Neuropsychology, 37(3), 226-252. https://doi.org/10.1080/87565641.2012.654867

Stewart, M.E., Ebmeier, K.P., \& Deary, I.J. (2005). Personality correlates of happiness and sadness: EPQ-R and TPQ compared. Personality and Individual Differences, 38,1085-1096. https://doi.org/10.1016/j.paid.2004.07.007

Uhlhaas, P.J., \& Singer, W. (2010). Abnormal neural oscillations and synchrony in schizophrenia. Nature Reviews Neuroscience, 11(2), 100-13. 
Figure 1.JPEG
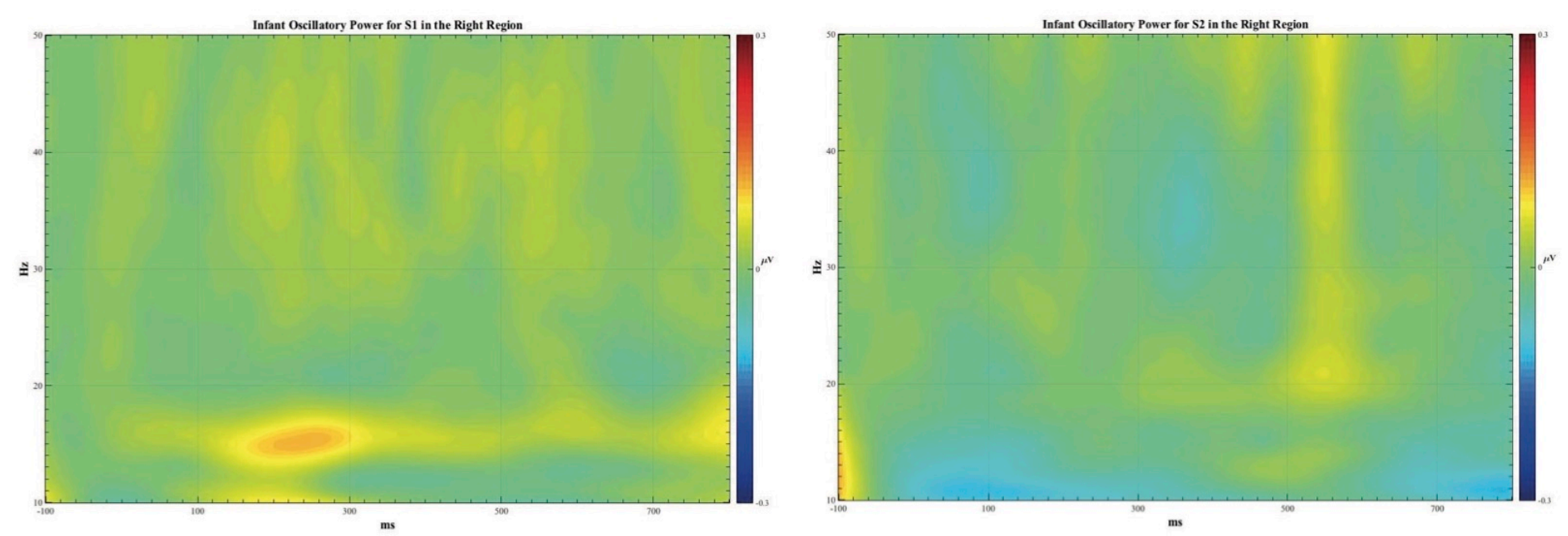
Figure 2.JPEG
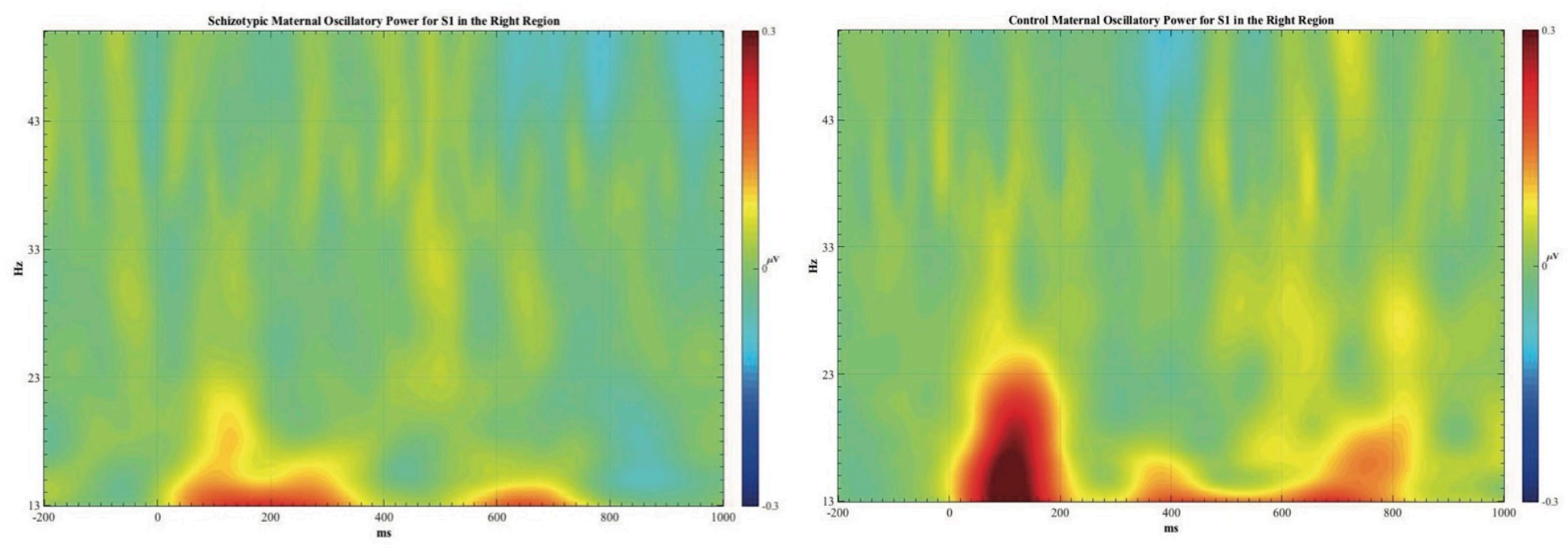
Figure 3.JPEG
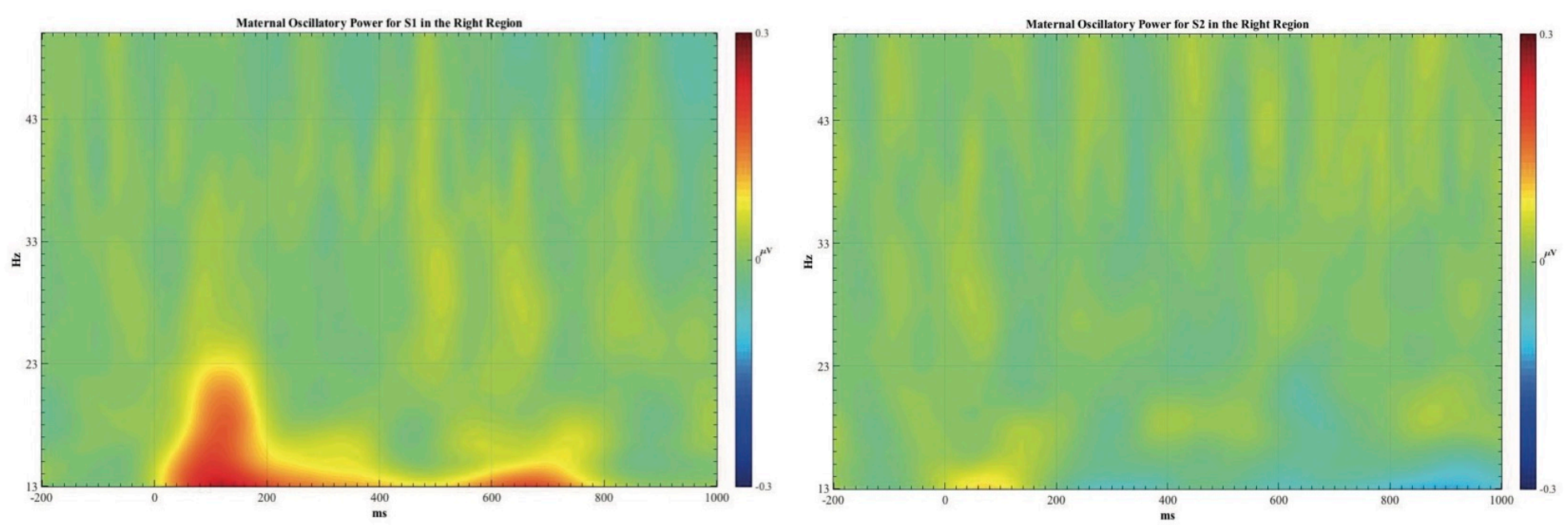

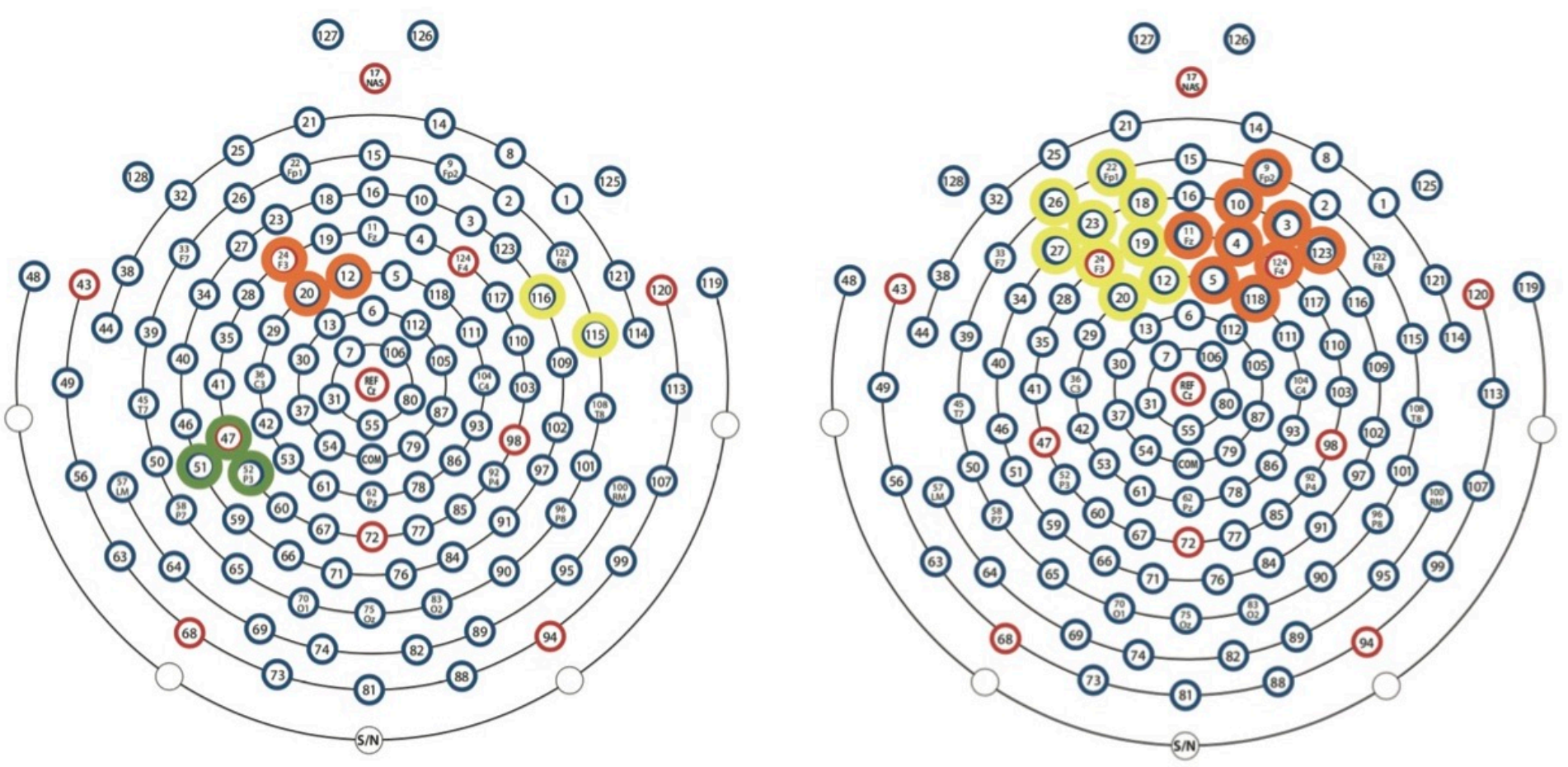


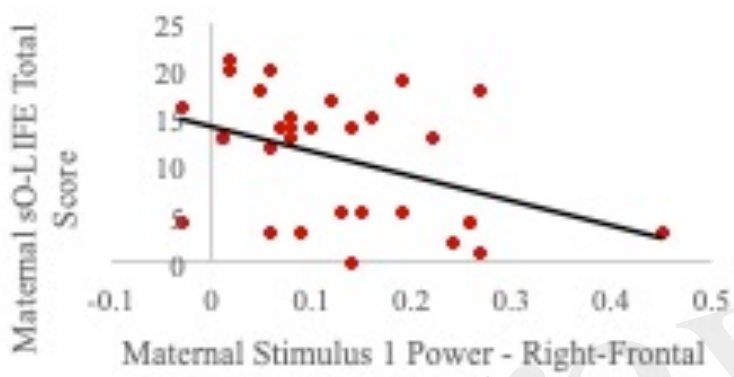
Region

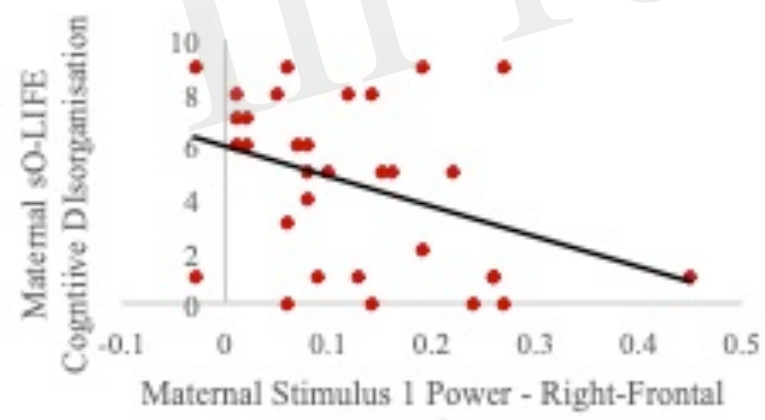

Region

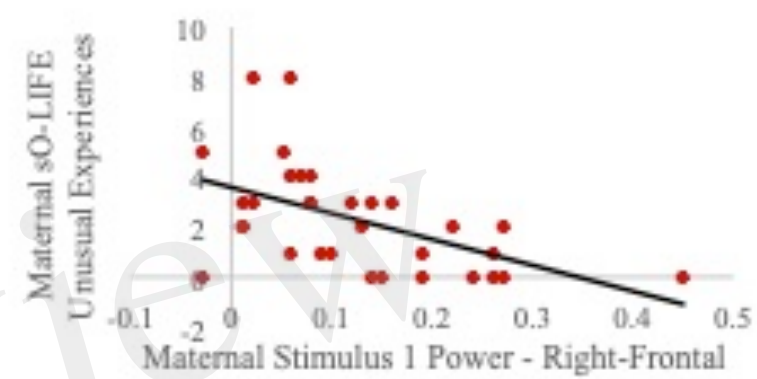

Region 
Stimulus 1

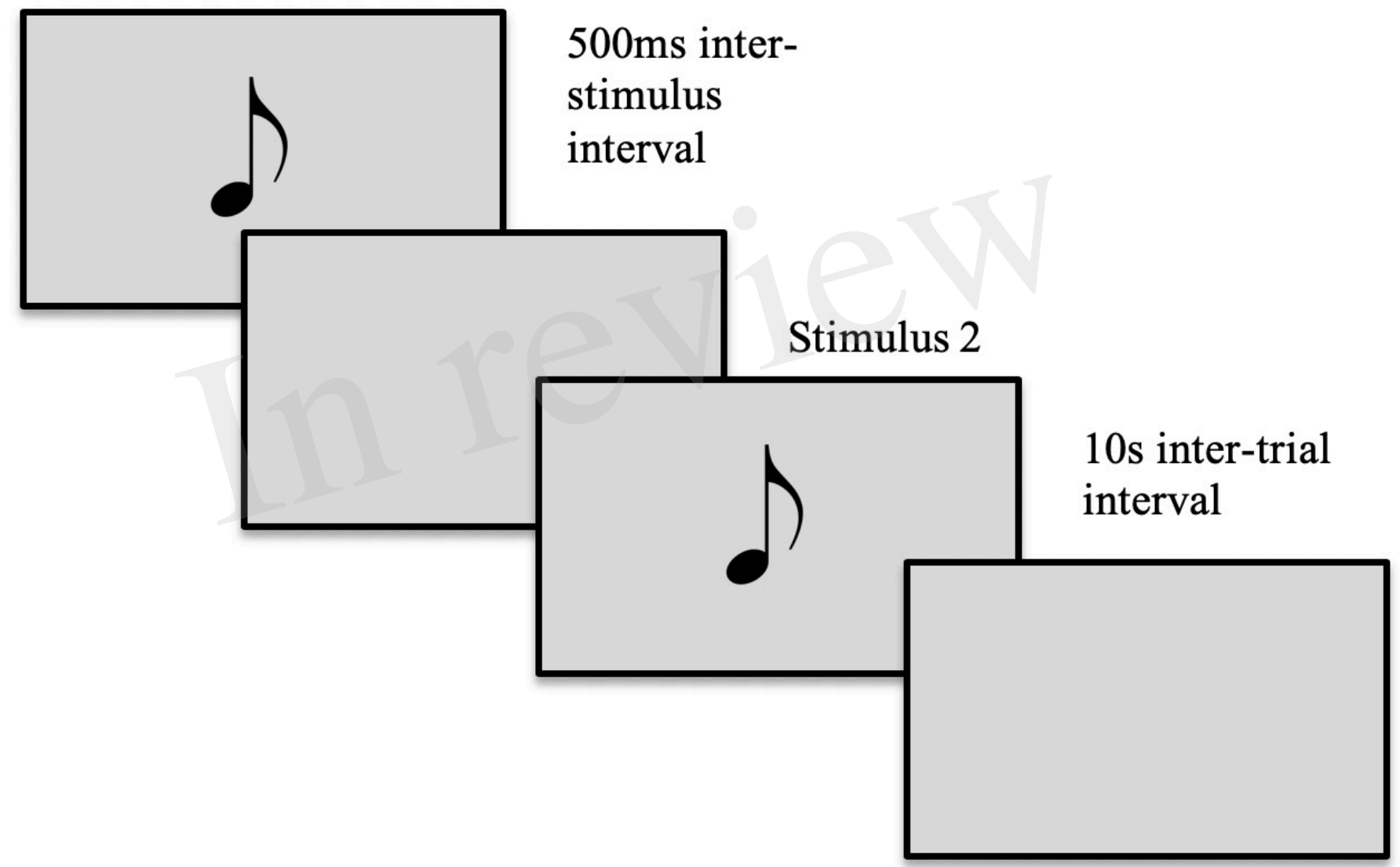

